Meta

Journal des tradlucteurs

Translators' Journal

\title{
Effets d'humour, ambiguïté et didactique de la traduction
}

\section{Michel Ballard}

Volume 34, numéro 1, mars 1989

Humour et traduction

Humour and Translation

URI : https://id.erudit.org/iderudit/001955ar

DOI : https://doi.org/10.7202/001955ar

Aller au sommaire du numéro

Éditeur(s)

Les Presses de l'Université de Montréal

ISSN

0026-0452 (imprimé)

1492-1421 (numérique)

Découvrir la revue

\section{Citer cet article}

Ballard, M. (1989). Effets d'humour, ambiguïté et didactique de la traduction. Meta, 34(1), 20-25. https://doi.org/10.7202/001955ar d'utilisation que vous pouvez consulter en ligne.

https://apropos.erudit.org/fr/usagers/politique-dutilisation/ 


\title{
EFFETS D'HUMOUR, AMBIGUÏTÉ ET DIDACTIQUE DE LA TRADUCTION
}

MiCHEL BALLARD

Université de Lille III, Lille, France

\begin{abstract}
[Un homme vient d'abattre un chien enragé d'un coup de bâton.] He dealt the prostrate brute two more blows which settled its fate. (N. West)

- Il acheva la brute en deux souffles qui installèrent son destin.
\end{abstract}

La traduction pourrait, selon Antoine Culioli, être décrite comme «un cas particulier de paraphrase avec un certain nombre de problèmes spécifiques» (Transcription du séminaire de D.E.A. 1975-1976, p. 29). C'est sans doute l'une des raisons pour lesquelles cette opération, lorsqu'elle est pratiquée comme exercice pédagogique, donne parfois lieu à d'étranges productions, dont certaines tombent dans la catégorie de l'humour. Il y a un élément de perte ou de déformation dans toute situation de communication et l'on connaît ces jeux où l'on propose à des enfants de faire circuler un message de bouche à oreille, en sachant bien qu'au bout de la chaîne il arrivera déformé de façon comique par rapport à l'original. Il devrait y avoir quelque chose de navrant dans l'erreur pour le pédagogue parce qu'elle est la preuve d'un échec ou d'une ignorance. Or, si certaines fautes irritent, il en est d'autres qui forcent le sourire ou le rire parce que leur écart par rapport à la norme tombe dans les schémas de l'humour verbal. On ne dérape pas sur du texte tout à fait au hasard, du moins pas toujours, les structures langagières sont parfois là pour aider l'apprenti traducteur à s'égarer avec une certaine logique. Celle de l'ambiguïté est sans doute l'une des plus productives, elle est en tout cas au cœur d'une problématique de la traduction puisqu'il s'agit de la perception du sens et de sa formulation.

Notre étude porte sur la traduction de l'anglais au français, pratiquée par des étudiants francophones en première année d'université. Il s'agit de prévenir (en partie selon le principe de l'homéopathie) des erreurs de manière organisée et de montrer que cette organisation constitue une gestion d'un bilinguisme encore mal assuré et donc déséquilibré. Les traductions fautives, à effet humoristique, sont précédées d'un astérisque; les traductions qui se voudraient correctes sont précédées d'une flèche. Nous restituons parfois le contexte nécessaire à la compréhension de la phrase en tête de celle-ci et entre crochets. Les segments concernés par le jeu de l'ambiguïté sont en gras dans le texte.

Tout message à traduire est d'abord un message à interpréter. Il y a ambiguïté d'un terme ou d'une structure lorsque ceux-ci peuvent offrir deux ou plusieurs sens au lecteur. Afin de décrire cette propriété et ses effets en traduction, il faut faire intervenir plusieurs paramètres: le matériau linguistique, la situation d'énonciation, les personnes impliquées dans l'acte langagier (l'émetteur, le premier récepteur qu'est le traducteur, le second récepteur qu'est l'auditeur ou le lecteur).

Le langage comporte dans sa texture des éléments qui représentent un potentiel d'ambiguité. Il y a toujours une composante syntagmatique dans la perception de l'ambiguîté, puisque, en dehors de situations métalinguistiques particulières (telle que la consultation des entrées d'un dictionnaire) notre saisie des mots s'effectue à travers des énoncés. Cependant nous distinguerons entre: $1^{\circ}$ le cas où l'ambiguîté a sa source dans 
un paradigme, et $2^{\circ}$, le cas où c'est la composante syntagmatique ou relationnelle qui prédomine (anaphore et syntaxe). Ce champ étant balisé, nous allons organiser notre étude autour de deux pôles: les signes pleins, les signes vides (et les mots-outils), nous ne traiterons qu'accessoirement de syntaxe.

L'actualisation du potentiel d'ambiguïté dans le texte peut être le fait de l'énonciateur. Il utilise la fonction ludique du langage qu'il inscrit de façon plus ou moins allusive dans le texte. Il y a là un effet à préserver par le traducteur, et celui-ci ne le fera que si, premièrement, il a perçu le jeu de l'ambigu à travers les formes présentes et, deuxièmement, s'il trouve dans la langue d'arrivée, et dans sa propre compétence, les moyens de transférer le rapport ambigu présent dans le texte.

L'actualisation du potentiel d'ambiguité peut être le fait du seul traducteur, elle révèle une mauvaise lecture qui s'inscrit dans le texte d'arrivée sous la forme d'un fauxsens. C'est lorsqu'elles versent dans le non-sens, l'absurde, le grotesque ou l'équivoque, que ces créations de l'incompétence produisent un effet d'humour dont nous étudierons deux axes de formation.

\section{HUMOUR ET PARADIGME D'AMBIGUITÉ}

L'ambiguïté sémiotique a sa source dans les ressemblances partielles ou totales entre signifiants (homophonie, homographie, paronymie) ou dans la profondeur du signifié (polysémie). Ces ressemblances et cette profondeur constituent le paradigme d'ambiguîté. Toute séquence du texte de départ susceptible d'être mise en relation avec un ou des éléments de ce paradigme constitue une source potentielle d'humour. Il convient par ailleurs de souligner que la spécificité de l'opération traduisante multiplie par deux le paradigme d'ambiguité puisque aux ressemblances intralinguistiques s'ajoutent les phénomènes d'interférence entre faux-amis.

\section{1. À PARTIR DE L'HOMOGRAPHIE}

- Effet d'humour par inversion du sens:

\section{He stroked his dog}

- Il donna un coup à son chien

Cette substitution d'une conduite hostile à un geste de sympathie est de toute évidence la manifestation d'une connaissance parcellaire de la langue, l'étudiant ne connaît que le sens du nom «stroke»; l'erreur se situe dans le champ d'une homographie intercatégorielle.

- Effet d'humour par création de l'irréel

[Il s'agit d'une poursuite dans le brouillard]

He ran forward and back, felt his heart clutched by a sickening fear, the dark fear that lives in the wings of fog. (J. Galsworthy)

- Il courait en avant et en arrière, et sentait son cour saisi par une peur qui le rendait malade, la peur noire qui vit dans les ailes du brouillard.

Cet effet est créé par la mise en rapport de deux termes qui habituellement n'entrent pas en collocation. L'incompatibilité sémantique entre les deux termes n'est pas totale si l'on y regarde bien, mais elle est ici marquée par le contexte. L'erreur dans ce cas est en partie due à une mauvaise lecture de la distribution de wings («coulisses») par rapport à wing («aile»). 
Il partit en courant et revint sur ses pas, une peur angoissante lui étreignit le cœur, la crainte obscure qui hante les profondeurs du brouillard.

Effet d'humour et jeu de mots

«My father's in the Navy. He said there aren't any unknown islands left.» (W. Golding)

- «Mon père est dans la Marine. Il a dit qu'il n'y avait aucune île à gauche.»

Le sens ainsi dégagé n'est pas totalement inacceptable (il suppose un contexte différent), et les constituants du paradigme d'homographie sont indéniablement connus de l'étudiant; il y a cependant une mauvaise lecture de la distribution du terme anglais. L'absurdité de l'énoncé traduit provient du caractère péremptoire de l'assertion présentée comme une vérité d'ordre général instituant une sorte de vide cosmique par rapport à un repère fixe absent («à gauche» de quoi ?) ou bien alors réduit au seul locuteur («à gauche» de qui ?), auquel cas le repère acquiert une mobilité encore plus déroutante.

«Mon père est dans la Marine. Il m'a dit que [littéralement : «il ne restait plus d'îles inconnues»] les îles inconnues, ça n'existe plus.»

\section{2 À PARTIR DE LA PARONYMIE}

La ressemblance entre les signifiants générateurs d'erreur n'est plus totale, elle se situe généralement au niveau de la racine.

Paronymie intralinguistique:

[le sujet représenté par «his» est un laveur de carreaux.]

Only his pail and his various cloths were of the finest quality that money could buy. (J. Wain)

- Seuls son chapeau et ses différents vêtements étaient de très grande qualité.

Seuls son seau et ses différents chiffons étaient de première qualité.

On est ici confronté à la classique confusion entre cloths et clothes. Mais il y a en plus un phénomène de lecture interférentielle interlinguistique à l'endroit de pail qui renvoie à «paille» perçu (par quels étranges circuits) comme une troncation du syntagme «chapeau de paille».

Paronymie interlinguistique

Elle se manifeste par la substitution au sens d'un mot anglais de celui d'un mot français qui lui ressemble mais qui n'appartient pas au même champ sémantique. Dans l'exemple suivant, l'effet d'humour est bien entendu couronné par la référence sexuelle en français. L'erreur lexicale rayonne sur la lecture de syntagme: the prostrate brute, dont la hiérarchie est inversée:

[ The prostrate brute» désigne le chien féroce que le héros «he», vient d'abattre d'un coup de bâton.]

He dealt the prostrate brute two more blows which settled its fate. (N. West)

- Il donna deux autres coups sur la prostate de la brute qui lui réglèrent son compte.

Il assena deux coups supplémentaires à la brute prostrée, ce qui lui régla son compte. 


\section{3 À PARTIR DES CONSTITUANTS}

Perfide, on le sait, l'anglais nous trompe même dans le sens global qu'il donne à certains composés non déductibles de leurs éléments constituants. Le comique ici est aussi un comique de situation, ou de posture.

At the gate she caught hold of my hand and gave it one squeeze and fell back. (J. Conrad)

- À la porte elle saisit ma main et la serra très fort et tomba à la renverse.

À la porte elle me saisit la main et la serra une seule fois, puis recula d'un pas. (H. Brodenave)

\section{4 À PARTIR DE LA POLYSÉMIE}

«If you must pay these calls, I will go with you.» (E. Gaskell)

- S'il faut que tu paies ces appels téléphoniques, j'irai avec toi.

Le décalage est ici en partie révélateur de l'ignorance du paramètre historique.

S'il faut que tu fasses ces visites, j'irai avec toi.

\subsection{L'INTERFÉRENCE SÉMTOTIQUE}

C'est le phénomène des faux-amis, homographes ou paronymes des deux langues qui n'ont pas le même signifié.

Our hero's way home led through a path that ran along the Rat river. As he passed a wooded stretch he cut a stout stick... He was twirling his club when he was startled by a young girl's shriek. Turning his head, he saw a terrified figure pursued by a fierce dog. (N. West)

Pour rentrer chez lui, notre héros devait emprunter un sentier qui longeait la rivière Rat. En traversant un bosquet, il se tailla un gros bâton... Il faisait des moulinets avec son gourdin quand le cri perçant d'une jeune fille le fit sursauter.

- Tournant la tête, il vit un visage terrifié poursuivi par un chien féroce.

Outre l'ignorance du lexique, cet effet d'humour surréaliste révèle une lecture syntagmatique étroite : la collocation avec terrified en anglais encourage le faux sens en français, puisqu'un «visage» peut être «terrifié», mais le rapport supérieur : «visage poursuivi par un chien» n'est pas acceptable. La traduction a d'ailleurs besoin de se dégager de l'équivalence figure — «silhouette, forme», à nouveau pour des raisons de collocation, l'adjectif «terrifié» ne peut s'appliquer à «silhouette». Le syntagme se réalise de façon acceptable par une recherche suivant les voies de la synecdoque : de la partie au tout.

Tournant la tête, il vit une personne terrifiée poursuivie par un chien féroce.

Bien qu'ils ne constituent pas directement notre objet ici, nous dirons un mot des noms propres car ils font bien apparaître la part de l'extralinguistique dans les jugements que nous portons sur les signes. Les noms propres ne devraient en principe pas poser de problème d'interprétation autre que celui de leur éventuelle définition puisqu'en théorie leur référent est unique. Or les erreurs constatées à leur sujet font bien ressortir à quel point l'interprétation repose sur un rapport que lecteur-traducteur doit établir entre le signe et une culture, un environnement :

[Une mère rend visite à son fils, étudiant, qui loge en meublé; elle lui fait quelques remarques.] 
"And I think you ought to lock that door at night»

"The people upstairs come in and out at all hours»

"Why can't they use keys like ordinary Christians?»

«They aren't Christians, they' re Muslims. And they would lose their keys and knock us up.»

(I. Murdoch)

L'erreur rencontrée dans une copie, au demeurant très bonne, a consisté à transformer les noms propres ici rattachés à des notions religieuses et civilisationnelles en patronymes du type: «Dupont» et «Dupond» dont l'opposition dans la phrase par sa futilité et sa loufoquerie ne fait qu'ajouter au comique de l'erreur d'interprétation:

- Pourquoi ne peuvent-ils utiliser leurs clés comme les Christians?

- Ce ne sont pas les Christians, ce sont les Muslims.

- Et alors! Ils ne peuvent pas avoir une clé comme tout le monde ?

- Ils ne sont pas comme tout le monde, ce sont des immigrés.

\section{HUMOUR ET ANAPHORE}

L'anaphore représente un potentiel d'ambiguïté en traduction, en particulier pour les débutants, surtout en ce qui a trait aux pronoms et adjectifs de rapport. L'existence en anglais de trois genres grammaticaux différenciés, permet une référence plus fine ou plus souple à des éléments de l'avant-texte.

Il y a des situations, où pour des raisons sémantiques ou syntaxiques, l'ambigu n'est qu'un potentiel :

Between eleven and twelve the garden gate clicked, and she lifted her eyes to the window. (Th. Hardy)

- Entre onze heures et midi la barrière du jardin claqua, et elle leva les yeux vers la fenêtre.

Il n'empêche que le fonctionnement de l'anaphore est tel que «la barrière» tend à s'interposer comme source sémantique entre «elle» et son véritable antécédent, en l'occurrence un personnage du nom de «Rhoda» dont le nom propre sera pris comme sujet grammatical en français.

Dans l'exemple suivant c'est la construction involontaire d'un syntagme figé à anaphore vague qui crée l'humour en français:

[la phrase suivante est une indication scénique]

Kate walks slowly to the bedroom door, goes out, closes it. (H. Pinter)

- Kate se dirige lentement vers la porte de la chambre, sort et la ferme.

... sort et referme la porte derrière elle.

Enfin voici un extrait où un type d'anaphore fondé, comme en anglais, sur l'utilisation de l'adjectif possessif, crée, en raison de l'effacement du genre, une confusion totale sur la référence à la personne:

[Lors d'une distribution de prix.]

The girl was aware of dark reports affecting Mr. Walsh's reputation. She hazarded only a brief examination of his features, and looked at the applauding Buckland with alarm.

«His lectures are splendid» said her brother, emphatically.

"If I were going to be here next session, I should take them». (G. Gissing) 
Il y a co-référence de Buckland et de her brother, la traduction de her brother par «son frère» construit, par effacement du genre en français et par proximité avec Buckland, la référence au «frère de Buckland». Cette anaphore lexicale impropre est rectifiée par effacement du rapport à la personne et désignation du personnage par un terme n'évoquant pas de lien de famille:

La jeune fille savait qu'il courait des bruits fâcheux sur la réputation de $M$. Walsh. Elle ne se hasarda que brièvement à examiner ses traits, et regarda avec inquiétude Buckland qui applaudissait. «Ses conférences sont remarquables, déclara le jeune homme avec force. Si j'étais ici l'an prochain, je m'y inscrirais».

L'humour est une forme de détachement par rapport au réel, qui permet d'en donner une présentation inattendue et amusante. En traduction, la référence est à construire à partir du texte, de la connaissance de deux langues et du jugement. L'humour traductologique involontaire se construit sur deux lignes de faille : des connaissances très fragmentaires et inorganisées, une forme de schizophrénie induite par un bilinguisme mal intégré. La traduction alors peut être une forme de thérapie, une manière de découvrir et de comprendre l'étrange par rapport à son propre système de représentation. La réponse à cet humour accidentel devrait être de donner les moyens de le maîtriser, c'est-à-dire une exploration systématique du lexique, du langage, de leurs structures, de leurs combinaisons et des jeux qu'ils permettent. Mais tout en s'efforçant d'être une initiation, un éveil à la profondeur et à la souplesse du langage, la didactique devra faire preuve de patience et d'humour pour supporter l'image que l'apprenant lui renverra parfois de sa propre démarche. Voici la forme sous laquelle nous est revenue la définition de l'homographie:

«Les homographes sont des mots de même graphie mais dont les sens respectueux n'entretiennent pas de rapport sémantique.»

\section{BIBLIOGRAPHIE}

BALLARD, Michel (1987) : la Traduction : de l'anglais au français, Paris, Nathan. BENVENISTE, Émile (1966) : Problèmes de linguistique générale, vol. I, Paris, Gallimard.

COLLECTIF (1984) : la Traduction: de la théorie à la didactique (textes de M. Ballard, M.Cl. et G. Bourquin,

G. Garnier, M. Krzak, J.R. Ladmiral, G. Mounin, M. Pergnier, Cl. Tatilon), Lille, P.U.L.

CULIOLI, Antoine : Transcription du séminaire de D.E.A. 1975-1976, D.R.L., Paris VII

GUIRAUD, Pierre (1979) : les Jeux de mots, Paris, P.U.F.

LANDHEER, Ronald (1984) : Aspects linguistiques et pragmatico-rhétoriques de l'ambiguité, thèse, Leiden. MITTERAND, Henri (1963) : les Mots français, Paris, P.U.F

PICOCHE, Jacqueline (1977): Précis de lexicologie française, Paris, Nathan.

QUIRK, Randolph, Sydney GREENBAUM (1973) : A University Grammar of English, London, Longman.

TESNIERE, Lucien (1959) : Éléments de syntaxe structurale, Paris, Klincksieck 